04

\title{
Особенности распределения неоднородного электростатического поля в системе с изменяемой конфигурацией электродов устройства, формирующего высоковольтный газовый разряд
}

\author{
(C) М.А. Маркушин, В.А. Колпаков, С.В. Кричевский \\ Самарский национальный исследовательский университет им. Акад. С.П. Королева, \\ 443086 Самара, Россия \\ e-mail: markushin_max@mail.ru
}

Поступило в Редакцию 17 января 2020 г.

В окончательной редакции 29 апреля 2020 г.

Принято к публикации 12 мая 2020 г.

\begin{abstract}
Предложена модель распределения неоднородного электростатического поля в системе с изменяемой конфигурацией электродов устройства, формирующего высоковольтный газовый разряд. Показана возможность применения метода конформного отображения функции комплексной переменной для аналитического описания характера распределения силовых линий и эквипотенциалей поля, зависящего от диаметра круглого отверстия в аноде, его толщины и межэлектродного расстояния, изменяющихся в пределах $D=1-5 \mathrm{~mm}$, $\Delta h=0.5-5 \mathrm{~mm}, h=1.2-5 \mathrm{~mm}$ соответственно. Получены расчетные изображения электрического поля, позволяющие определить их связь с электрофизическими параметрами электродной системы устройства. На основе полученных результатов расчета дополняются и уточняются обширные экспериментальные данные, касающиеся исследования особенностей такого разряда.
\end{abstract}

Ключевые слова: низкотемпературная плазма, высоковольтный газовый разряд, конформное отображение, электростатическое поле, концентрация заряженных частиц.

DOI: $10.21883 /$ JTF.2020.12.50120.21-20

\section{Введение}

Высоковольтный газовый разряд представляет интерес как средство формирования направленных потоков низкотемпературной внеэлектродной плазмы, отличающейся своими оригинальными особенностями [1-7], и является средством формирования направленных потоков заряженных частиц низкотемпературной внеэлектродной плазмы, которая широко используется для изготовления омических контактов к полупроводниковым элементам, увеличения адгезии тонких металлических пленок, очистки, а также микро- и наноразмерного структурирования поверхности полупроводниковых и диэлектрических материалов $[1,2,8]$. В работах $[1-3,8,9]$ обоснованы преимущества такой плазмы перед низкотемпературной плазмой, формируемой современными источниками. В работе [10] представлены особенности кинетики заряженных частиц высоковольтного газового разряда в неоднородном электростатическом поле. Отмеченные преимущества и особенности определяются характером распределения электростатического поля в системе электродов устройства, конфигурация которой подробно рассматривалась в работах $[2,3,8-10]$. В работе [9] представлена модель распределения неоднородного электростатического поля такой электродной системы. Однако в данных работах отсутствуют сведения о зависимости концентрации заряженных частиц от геометрических параметров электродной системы (в частности, от толщины анода).
В связи с этим представляет интерес получение новых знаний об особенностях распределения неоднородного электростатического поля, определяемых геометрическими параметрами: диаметром круглого отверстия в аноде, его толщиной и межэлектродным расстоянием. Кроме того, с помощью разрабатываемой модели должны быть получены расчетные значения концентрации электронов в заданной точке. Эти данные позволят расширить имеющиеся теоретические знания о процессах, протекающих в газовых разрядах, существующих в неоднородных электрических полях, а также осуществить тщательный анализ влияния параметров электродной системы, формирующей высоковольтный газовый разряд, на электрофизические параметры внеэлектродной плазмы.

\section{1. Описание модели}

Основной трудностью нахождения аналитического описания распределения силовых линий и эквипотенциалей электростатического поля устройства, формирующего высоковольтный газовый разряд, является существенно неравномерный характер распределения этого поля за пределами межэлектродного расстояния [9]. В связи с этим поставленную задачу целесообразно свести к решению задачи двумерной, что позволяет значительно упростить процесс моделирования распределения исследуемого электростатического поля, использующий метод конформного отображения функции комплексного 


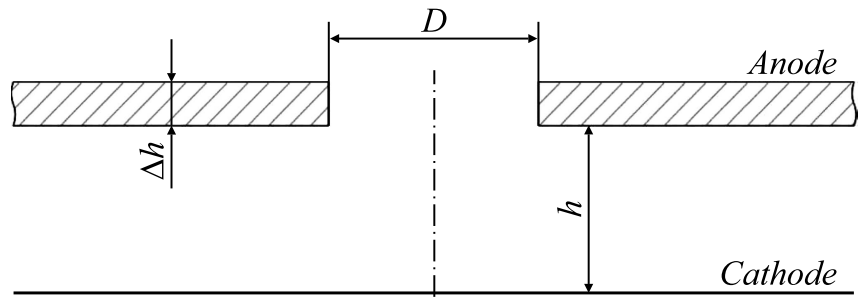

Pис. 1. Схематичное изображение электродной системы на основе элементарной ячейки.
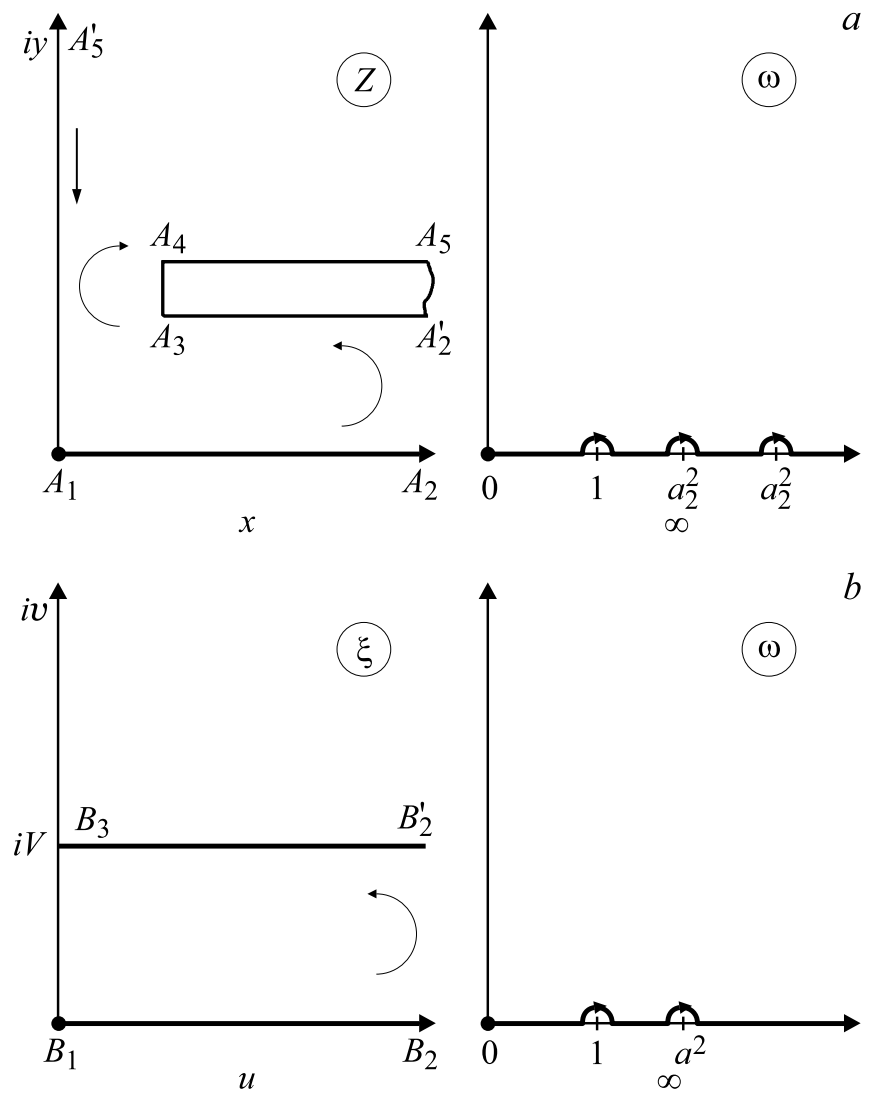

Рис. 2. Схема конформного отображения электродной системы газоразрядного устройства: $a-$ конформное отображение верхней полуплоскости $\omega$ на область поля $Z$ с электродами; $b$ - дополнительное конформное отображение полуплоскости $\omega$ на полосу $0<\operatorname{Im} \xi<V$.

переменного. Поскольку межэлектродное пространство за пределами области отверстия в аноде представляет собой конденсатор с равномерным распределением поля, конструкцию электродной системы целесообразно моделировать бесконечно удаленными электродами с отверстием в аноде (рис. 1).

В связи с тем, что геометрические параметры электродной системы оказывают влияние на распределение поля, возникает необходимость учитывать их при расчетах. Значения таких параметров для численного эксперимента были выбраны следующие: $D=1-5 \mathrm{~mm}$, $\Delta h=0.5-5 \mathrm{~mm}, h=1.2-5 \mathrm{~mm}$.

В силу симметрии электродной системы, при конформном отображении можно рассматривать только правую ее часть на комплексной плоскости $Z[11,12]$. В результате имеем многоугольник $A_{1} A_{2} A_{3} A_{4} A_{5}$ (рис. 2).

Моделирование, как и в работе [9], сводится к двум конформным отображениям: отображение $Z=f(\omega)$ верхней полуплоскости $\operatorname{Im} \omega>0$ на область поля $Z$ с электродами $A_{1} A_{2}$ (катод), $A_{3} A_{4} A_{5}$ (анод) (рис. 2,a), далее - дополнительное отображение $\xi=f(\omega)$ полуплоскости $\omega$ на полосу (рис. $2, b$ ), которое полностью совпадает с результатом конформного отображения второго этапа моделирования, представленного в работе [9]:

$$
\xi=\frac{2 V}{\pi} \operatorname{arcth} \sqrt{\omega},
$$

где $V$ - напряжение на электродах.

Цель двух конформных отображений: связать комплексный потенциал $\xi=u(x, y)+i v(x, y)$ с координатами $x$ и $y$ области электростатического поля $z$.

Для конформного отображения верхней полуплоскости $\omega$ на область поля $Z$ с электродами вершинам $A_{1} A_{2} A_{3} A_{4} A_{5}$ плоскости $Z$ ставятся в соответствие точки действительной оси плоскости $\omega$. Три граничные точки (например, 0, 1 и $\infty$ ), согласно теореме о единственности конформного отображения, можно задать произвольHо $[12,13]$ :

$\begin{array}{ccccc}A_{1} & A_{2} & A_{3} & A_{4} & A_{5} \\ \mathbb{1} & \mathbb{1} & \mathbb{1} & \mathbb{1} & \mathbb{1} \\ 0 & 1 & a_{1} & a_{2} & \infty .\end{array}$

Рассматривая внутреннюю область пятиугольника и двигаясь в положительном направлении обхода ее границы, по методике [11-13] определяем углы $\mu_{k}$, дополняющие внутренние углы при вершинах пятиугольника $A_{1} A_{2} A_{3} A_{4} A_{5}$, до $\pi$ : $\mu_{1}=1 / 2 ; \mu_{2}=1 ; \mu_{3}=-1 / 2$; $\mu_{4}=-1 / 2 ; \mu_{5}=3 / 2$. Выполнение равенства $\sum_{i=1}^{5} \mu_{i}=2$ подтверждает правильность полученных значений углов $\mu_{k}$ [11]. Подставляя углы $\mu_{k}$ в интеграл Шварца-Кристоффеля [12], находим отображающую функцию:

$$
\begin{aligned}
Z= & C \int_{0}^{\omega}\left(\omega-a_{1}\right)^{-\mu_{1}}\left(\omega-a_{2}\right)^{-\mu_{2}} \ldots\left(\omega-a_{n}\right)^{-\mu_{n}} d \omega+C_{1} \\
= & C \int_{0}^{\omega} \frac{\sqrt{\left(\omega-a_{1}\right)\left(\omega-a_{2}\right)}}{(\omega-1) \sqrt{\omega}} d \omega+C_{1} .
\end{aligned}
$$

Здесь $C$ - постоянный коэффициент, зависящий от геометрических параметров электродной системы: диаметра круглого отверстия в аноде, его толщины и межэлектродного расстояния. 
Решение данного интеграла осуществлено с помощью пакета Mathematica, тогда:

$$
\begin{aligned}
& Z=\frac{2 i}{\sqrt{a_{1}-a_{2}}} C\left[( a _ { 1 } - 1 ) F \left(i \sinh ^{-1}\left(\sqrt{\frac{\omega}{a_{1}}-1}\right) \mid\right.\right. \\
& \left.\times \frac{a_{1}}{a_{1}-a_{2}}\right)+\left(a_{2}-a_{1}\right) E\left(i \sinh ^{-1}\left(\sqrt{\frac{\omega}{a_{1}}-1}\right) \mid\right. \\
& \left.\times \frac{a_{1}}{a_{1}-a_{2}}\right)-\left(a_{2}-1\right) \Pi\left(\frac{a_{1}}{a_{1}-1} ; i \sinh ^{-1}\left(\sqrt{\frac{\omega}{a_{1}}-1}\right) \mid\right. \\
& \left.\left.\times \frac{a_{1}}{a_{1}-a_{2}}\right)\right]\left.\right|_{0} ^{\omega}+C_{1} .
\end{aligned}
$$

После подстановки пределов интегрирования

$$
\begin{aligned}
& Z=\frac{2 i}{\sqrt{a_{1}-a_{2}}} C\left[( a _ { 1 } - 1 ) \left[F \left(i \sinh ^{-1}\left(\sqrt{\frac{\omega}{a_{1}}-1}\right) \mid\right.\right.\right. \\
& \left.\left.+\frac{a_{1}}{a_{1}-a_{2}}\right)+F\left(\frac{a_{1}}{a_{1}-a_{2}}\right)\right]+\left(a_{2}-a_{1}\right) \\
& \times\left[E\left(i \sinh ^{-1}\left(\sqrt{\frac{\omega}{a_{1}}-1}\right) \mid \frac{a_{1}}{a_{1}-a_{2}}\right)+E\left(\frac{a_{1}}{a_{1}-a_{2}}\right)\right] \\
& -\left(a_{2}-1\right)\left[\Pi\left(\frac{a_{1}}{a_{1}-1} ; i \sinh ^{-1}\left(\sqrt{\frac{\omega}{a_{1}}-1}\right) \mid \frac{a_{1}}{a_{1}-a_{2}}\right)\right. \\
& \left.\left.+\Pi\left(\frac{a_{1}}{a_{1}-1} ; \frac{a_{1}}{a_{1}-a_{2}}\right)\right]\right]+C_{1} .
\end{aligned}
$$

Учитывая соответствие точек $A_{1}$ и $\omega=0$, получаем, что $C_{1}=0$.

Переход с нижнего электрода на верхний соответствует переходу луча $A_{1} A_{2}$ на луч $A_{2} A_{3}$ (рис. 2). В результате функция получает приращение

$$
\Delta Z=i h .
$$

С учетом того что при обходе точки $\omega=1$ аргумент меняется от $\pi$ до 0 , можно записать выражение

$$
\Delta Z=C(-i \pi) \sqrt{\left(1-a_{1}\right)\left(1-a_{2}\right)} .
$$

Тогда из (3) и (4) следует

$$
C=-\frac{h}{\pi \sqrt{\left(1-a_{1}\right)\left(1-a_{2}\right)}} .
$$

Из соответствия точек $A_{3}$ и $\omega=a_{1}$ получаем

$$
\begin{aligned}
& D+i h=\frac{2 i}{\sqrt{\left(a_{1}-a_{2}\right)}} C \\
& \times\left(\begin{array}{l}
\left(a_{1}-1\right) F\left(\frac{a_{1}}{a_{1}-a_{2}}\right)+\left(a_{1}-a_{2}\right) E\left(\frac{a_{1}}{a_{1}-a_{2}}\right) \\
-\left(a_{1}-1\right) \Pi\left(\frac{a_{1}}{a_{1}-1} ; \frac{a_{1}}{a_{1}-a_{2}}\right)
\end{array}\right) .
\end{aligned}
$$

Из соответствия точек $A_{4}$ и $\omega=a_{2}$ имеем

$$
\begin{aligned}
& D+i(h+\Delta h)=\frac{2 i}{\sqrt{a_{1-a_{2}}}} C\left[\left(a_{1}-1\right) F\right. \\
& \times\left(i \sinh ^{-1}\left(\sqrt{\frac{a_{2}}{a_{1}}-1}\right) \mid \frac{a_{1}}{a_{1}-a_{2}}\right)+\left(a_{2}-a_{1}\right) E \\
& \times\left(i \sinh ^{-1}\left(\sqrt{\frac{a_{2}}{a_{1}}-1}\right) \mid \frac{a_{1}}{a_{1}-a_{2}}\right)-\left(a_{2}-1\right) \Pi \\
& \left.\times\left(\frac{a_{1}}{a_{1}-1} ; i \sinh ^{-1}\left(\sqrt{\frac{a_{2}}{a_{1}}-1}\right) \mid \frac{a_{1}}{a_{1}-a_{2}}\right)\right] .
\end{aligned}
$$

Решая систему уравнений из равенств (5) и (6), получаем значения искомых точек $a_{1}$ и $a_{2}$ (рис. 2). Далее, подставив функцию (1) в уравнение (2), получаем модель неоднородного электростатического поля в системе c изменяемой конфигурацией электродов устройства, формирующего высоковольтный газовый разряд:

$$
\begin{aligned}
& Z=\frac{2 i}{\sqrt{a_{2}-a_{1}}} C\left[( a _ { 2 } - 1 ) \left[F \left(i \sinh ^{-1}\left(\sqrt{\left.\frac{t h^{2}\left(\frac{\xi \pi}{2 V}\right)}{a_{2}}-1\right)}\right) \mid\right.\right.\right. \\
& \left.\left.\times \frac{a_{2}}{a_{2}-a_{1}}\right)+F\left(\frac{a_{2}}{a_{2}-a_{1}}\right)\right]+\left(a_{1}-a_{2}\right)[E \\
& \left.\times\left(i \sinh ^{-1}\left(\sqrt{\frac{t h^{2}\left(\frac{\xi \pi}{2 V}\right)}{a_{2}}-1}\right) \mid \frac{a_{2}}{a_{2}-a_{1}}\right)+E\left(\frac{a_{2}}{a_{2}-a_{1}}\right)\right] \\
& -\left(a_{1}-1\right)\left[\Pi \left(\frac{a_{2}}{a_{2}-1} ; i \sinh ^{-1}\left(\sqrt{\left.\frac{t h^{2}\left(\frac{\xi \pi}{2 V}\right)}{a_{2}}-1\right)}\right)\right.\right. \\
& \left.\left.\times \frac{a_{2}}{a_{2}-a_{1}}\right)+\Pi\left(\frac{a_{2}}{a_{2}-a_{1}} ; \frac{a_{2}}{a_{2}-a_{1}}\right)\right]
\end{aligned}
$$

Напряженность электростатического поля определяется по формуле [11]

$$
E=-i \cdot \frac{1}{z^{\prime}(\bar{\xi})}
$$

Подстановка выражения (7) в (8) приводит к уравнению напряженности неоднородного электростатического 
поля в рассматриваемой электродной системе

$$
\begin{aligned}
& E=\frac{\pi \sqrt{\left(1-a_{1}\right)\left(1-a_{2}\right)} \sqrt{a_{1}-a_{2}} / 2 h}{i \pi\left(a_{1}-1\right) \operatorname{Sech}^{2}\left(\frac{\xi \pi}{2 V}\right) \operatorname{th}\left(\frac{\xi \pi}{2 V}\right)}- \\
& 2 V a_{1} \sqrt{\frac{\operatorname{th}^{2}\left(\frac{\xi \pi}{2 V}\right)}{a_{1}}} \sqrt{\frac{\operatorname{th}^{2}\left(\frac{\xi \pi}{2 V}\right)}{a_{1}}-1} \sqrt{1-\frac{a_{1}\left(1-\frac{\operatorname{th}^{2}\left(\frac{\xi \pi}{2 V}\right)}{a_{1}}\right)}{a_{1}-a_{2}}} \\
& -\frac{i \pi\left(a_{2}-1\right) \operatorname{Sech}^{2}\left(\frac{\xi \pi}{2 V}\right) \operatorname{th}\left(\frac{\xi \pi}{2 V}\right)}{2 V a_{1} \sqrt{\frac{\operatorname{th}^{2}\left(\frac{\xi \pi}{2 V}\right)}{a_{1}}} \sqrt{\frac{\operatorname{th}^{2}\left(\frac{\xi \pi}{2 V}\right)}{a_{1}}-1 \times}}+ \\
& \times\left(1-\frac{a_{1}\left(1-\frac{\operatorname{th}^{2}\left(\frac{\xi \pi}{2 V}\right)}{a_{1}}\right)}{a_{1}-1}\right) \sqrt{1-\frac{a_{1}\left(1-\frac{\mathrm{th}^{2}\left(\frac{\xi \pi}{2 V}\right)}{a_{1}}\right)}{a_{1}-a_{2}}} \\
& +\frac{i \pi\left(a_{2}-a_{1}\right) \operatorname{Sech}^{2}\left(\frac{\xi \pi}{2 V}\right) \operatorname{th}\left(\frac{\xi \pi}{2 V}\right) \sqrt{1-\frac{a_{1}\left(1-\frac{\mathrm{th}^{2}\left(\frac{\xi \pi}{2 V}\right)}{a_{1}}\right)}{a_{1}-a_{2}}}}{2 V a_{1} \sqrt{\frac{\mathrm{th}^{2}\left(\frac{\xi \pi}{2 V}\right)}{a_{1}}} \sqrt{\frac{\mathrm{th}^{2}\left(\frac{\xi \pi}{2 V}\right)}{a_{1}}-1}} .
\end{aligned}
$$

Таким образом, подставляя в выражения (5)-(7) и (9) соответствующие значения параметров $h, \Delta h, V$, $D$, можно расчетным путем получить распределение неоднородного электростатического поля в электродной системе, формирующей высоковольтный газовый разряд, с необходимым значением напряженности в заданной точке внеэлектродного пространства.

\section{2. Результаты расчетов}

Расчеты проводились с использованием полученных выражений (7) и (9) при давлении газа $p=0.15$ Torr, его температуре $T=293 \mathrm{~K}$, напряжении на электродах $V=1200 \mathrm{~V}$ и токе разряда $I=0.12 \mathrm{~A}$. Первые две величины использовались для расчета концентрации молекул остаточного газа, от которой, в свою очередь, зависит длина свободного пробега заряженных частиц. Задаваясь толщиной анода $\Delta h$, межэлектродным расстоянием $h$ и радиусом отверстия в аноде $D$, можно получить картины распределения электростатического поля, которое зависит от вышеуказанных параметров.

Варьируя толщину анода при постоянных значениях остальных конструктивных параметров, получаем распределения поля, показанные на рис. 3.

Как можно заметить, увеличение толщины анода приводит к „просаживанию“ эквипотенциалей поля и уменьшению длины прямолинейных участков силовых линий.

Влияние диаметра отверстия в аноде и межэлектродного расстояния на распределение электростатического поля было рассмотрено в работе [14]. Судя по полученным в вышеупомянутой работе распределениям, увеличение диаметра отверстия в аноде $D$ приводит к увеличению длины прямолинейных участков силовых линий, что хорошо просматривается на рис. 4 (для наглядности силовые линии при разных значениях величины $D$ были построены выходящими из одной точки на плоскости катода).

При увеличении межэлектродного расстояния длина прямолинейных участков силовых линий также будет увеличиваться [14], но эквипотенциали электростатического поля практически не будут выходить за пределы отверстия в аноде.

Так как нас интересует плазма, формирующаяся именно за пределами электродов, полученные результаты имеют ценность при выборе конструктивных параметров, которые будут определять необходимое распределение электростатического поля. Кроме того, эти параметры прямым образом влияют на концентрацию заряженных частиц плазмы.

С целью анализа влияния конструктивных параметров на концентрацию электронов внеэлектродной низкотемпературной плазмы и напряженность электростатического поля выберем точку $(x=0, y=14.25)$ в области плазмы, где, согласно расчетам [10], происходит первый акт ионизации.

Концентрацию электронов найдем, используя формулу [15]:

$$
n_{e}=\frac{j m_{e} \cdot 10^{-5}}{e^{2} E t}
$$

где $j=\frac{I}{\eta S}\left[\mathrm{~A} / \mathrm{cm}^{2}\right]-$ плотность тока; $I=0.12-$ ток разряда $[\mathrm{A}] ; \eta=0.95-$ коэффициент прозрачности анода; $S$ - площадь катода $78.5 \mathrm{~cm}^{2} ; m_{e}-$ масса электрона; $e-$ элементарный заряд; $t=7 \cdot 10^{-10} \mathrm{~s}-$ время свободного пробега электрона, рассчитанное по методике [10].

В таблице приведены расчетные данные по напряженности электростатического поля и концентрации электронов в локальной точке на оси отверстия в аноде в зависимости от конструктивных параметров электродной системы.

Согласно результатам расчетов, при толщине анода более $5 \mathrm{~mm}$ эквипотенциали поля практически не выходят за пределы отверстия в аноде и, несмотря на увеличение при этом концентрации электронов в локальной точке, равномерность распределения плотности заряженных частиц по сечению плазмы будет значительно уменьшаться. С другой стороны, высокой равномерности можно добиться, если увеличивать размер ячейки анода, однако это, в свою очередь, приведет к снижению концентрации электронов.

Таким образом, уменьшение межэлектродного расстояния и увеличение диаметра ячейки в аноде приводит к увеличению напряженности электростатического поля и, как следствие, к уменьшению концентрации электронов в локальной точке пространства. Обратный эффект наблюдаем при увеличении толщины анода.

Объяснить влияние напряженности поля на концентрацию электронов можно следующим образом. Электроны в момент выхода за пределы отверстия в аноде об- 

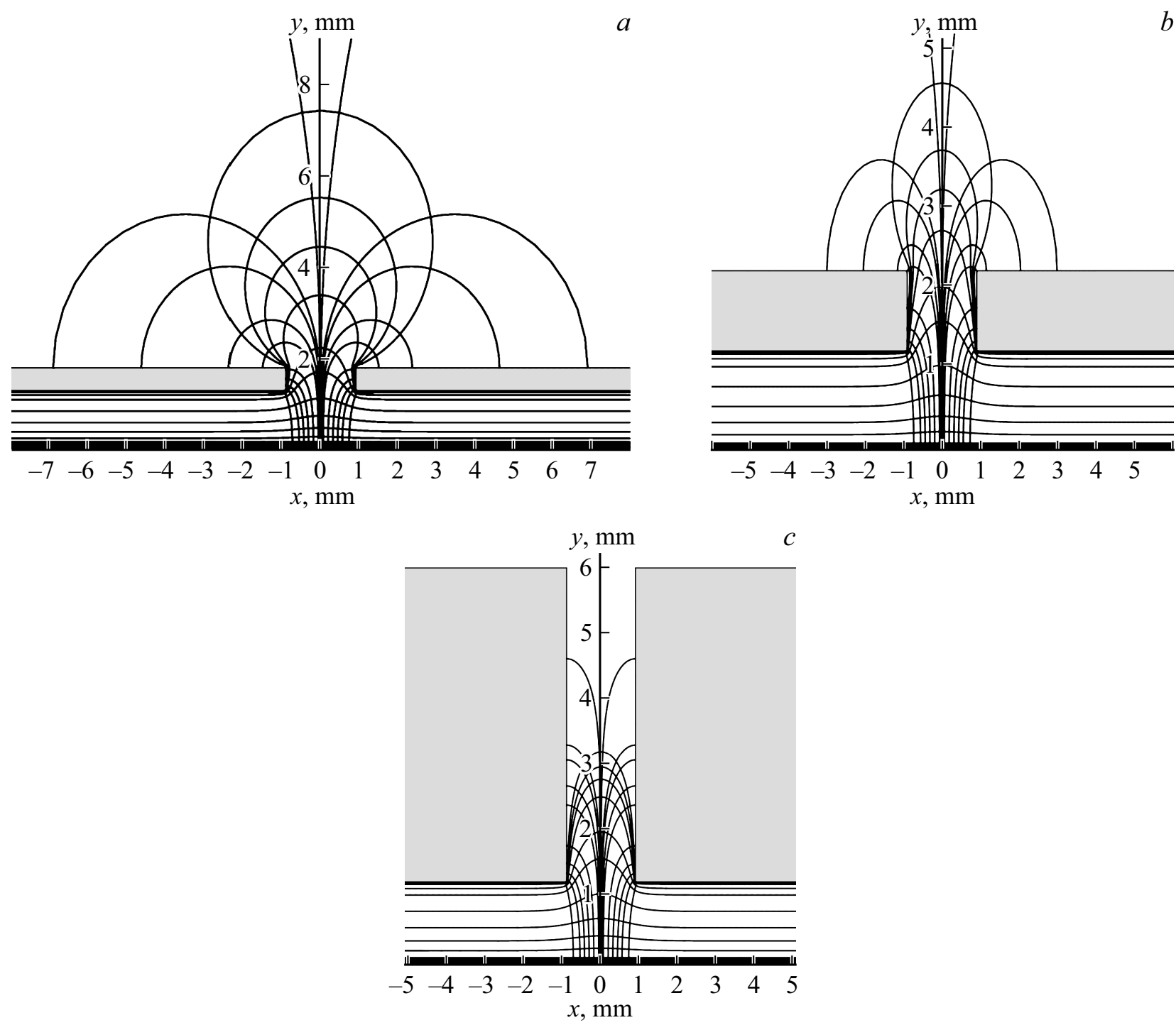

Рис. 3. Распределение электростатического поля при $D=1.8 \mathrm{~mm}, h=1.2 \mathrm{~mm}: a-\Delta h=0.5 ; b-1 ; c-4.8 \mathrm{~mm}$.

Результаты расчетов концентрации электронов $\left(\mathrm{cm}^{-3}\right)$ и напряженности электростатического поля $(\mathrm{V} / \mathrm{mm})$

\begin{tabular}{|c|c|c|c|c|}
\hline $\begin{array}{c}x=0 \mathrm{~mm} \\
y=14.25 \mathrm{~mm}\end{array}$ & $\begin{array}{c}h=1.2 \mathrm{~mm} \\
\Delta h=0.5 \mathrm{~mm}\end{array}$ & $\begin{array}{l}h=1.2 \mathrm{~mm} \\
\Delta h=1 \mathrm{~mm}\end{array}$ & $\begin{array}{c}h=2.7 \mathrm{~mm} \\
\Delta h=0.5 \mathrm{~mm}\end{array}$ & $\begin{array}{l}h=2.7 \mathrm{~mm} \\
\Delta h=1 \mathrm{~mm}\end{array}$ \\
\hline$D=1 \mathrm{~mm}$ & $\begin{array}{c}n_{e}=7.30572 \cdot 10^{9} \\
E=0.111848\end{array}$ & $\begin{array}{c}n_{e}=3.35203 \cdot 10^{10} \\
E=0.0243772\end{array}$ & $\begin{array}{c}n_{e}=1.25299 \cdot 10^{10} \\
E=0.0652144\end{array}$ & $\begin{array}{c}n_{e}=5.3909 \cdot 10^{10} \\
E=0.0151576\end{array}$ \\
\hline$D=1.8 \mathrm{~mm}$ & $\begin{array}{c}n_{e}=1.267461 \cdot 10^{9} \\
E=0.644698\end{array}$ & $\begin{array}{c}n_{e}=2.70025 \cdot 10^{9} \\
E=0.302613\end{array}$ & $\begin{array}{c}n_{e}=1.93954 \cdot 10^{9} \\
E=0.421301\end{array}$ & $\begin{array}{c}n_{e}=4.18083 \cdot 10^{9} \\
E=0.195447\end{array}$ \\
\hline$D=3 \mathrm{~mm}$ & $\begin{array}{c}n_{e}=3.58174 \cdot 10^{8} \\
E=2.28138\end{array}$ & $\begin{array}{c}n_{e}=5.50275 \cdot 10^{8} \\
E=1.48495\end{array}$ & $\begin{array}{c}n_{e}=5.03739 \cdot 10^{8} \\
E=1.62213\end{array}$ & $\begin{array}{c}n_{e}=7.7587 \cdot 10^{8} \\
E=1.05318\end{array}$ \\
\hline$D=5 \mathrm{~mm}$ & $\begin{array}{c}n_{e}=1.28026 \cdot 10^{8} \\
E=6.38251\end{array}$ & $\begin{array}{c}n_{e}=1.61041 \cdot 10^{8} \\
E=5.07406\end{array}$ & $\begin{array}{c}n_{e}=1.61841 \cdot 10^{8} \\
E=5.04896\end{array}$ & $\begin{array}{c}n_{e}=2.01106 \cdot 10^{8} \\
E=4.06318\end{array}$ \\
\hline
\end{tabular}


ладают энергией более $0.66 \mathrm{eV}$. В связи с этим, исходя из графика зависимости сечения взаимодействия электрона с нейтральными молекулами остаточного газа $[15,16]$ и следующего выражения [15]:

$$
v_{d}=\mu_{e} E,
$$

где $v_{d}-$ скорость дрейфа электронов; $\mu_{e}-$ подвижность электронов, получаем, что чем больше напряженность, тем выше скорость электронов и соответственно меньше вероятность ионизации атома/молекулы остаточного газа $[15,16]$.

Стоит также отметить, что чем меньше величина $\Delta h$ и больше $D$, тем больше область объемной ионизации атомов остаточного газа электронами. Следствием этого является увеличение рабочей площади на катоде, вызванное бомбардировкой положительными ионами (рис. 5).

Экспериментальные данные, полученные однозондовым методом в вакуумной камере при тех же значениях параметров (давление, напряжение, ток разряда, температура газа, конструктивные параметры электродной системы), что используются в модели, свидетельствуют о ее адекватности. Эксперимент, проведенный при значениях $D=5 \mathrm{~mm}, h=1.2 \mathrm{~mm}, \Delta h=1 \mathrm{~mm}$, позволил определить концентрацию электронов [17] в контрольной точке: $n_{e}=3.6 \cdot 10^{8} \mathrm{~cm}^{-3}$. Результатом моделирования при тех же условиях стало значение $n_{e}=1.61 \cdot 10^{8} \mathrm{~cm}^{-3}$.

Кроме того, из таблицы видно, что при диаметре ячейки $D=1 \mathrm{~mm}$, концентрация электронов имеет порядок $10^{10} \mathrm{~cm}^{-3}$. При такой концентрации радиус Дебая, вычисляемый по формуле [15]:

$$
r_{D}=6.90 \sqrt{\frac{T_{e}}{n_{e}}},
$$

где $T_{e}=2 \cdot 10^{4} \mathrm{~K}$ - электронная температура, вычисленная по методике [17], принимает значение

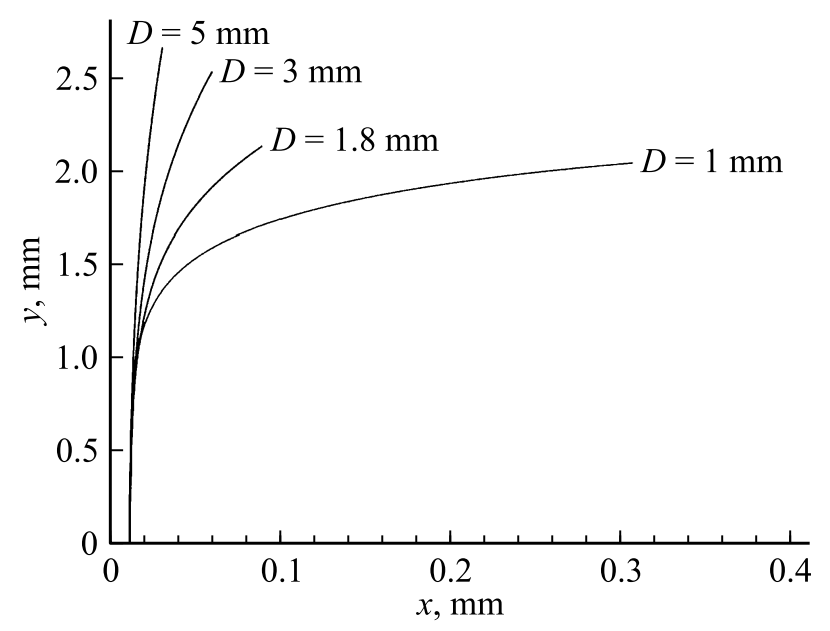

Рис. 4. Изображение участков силовых линий при разном радиусе отверстия в аноде.

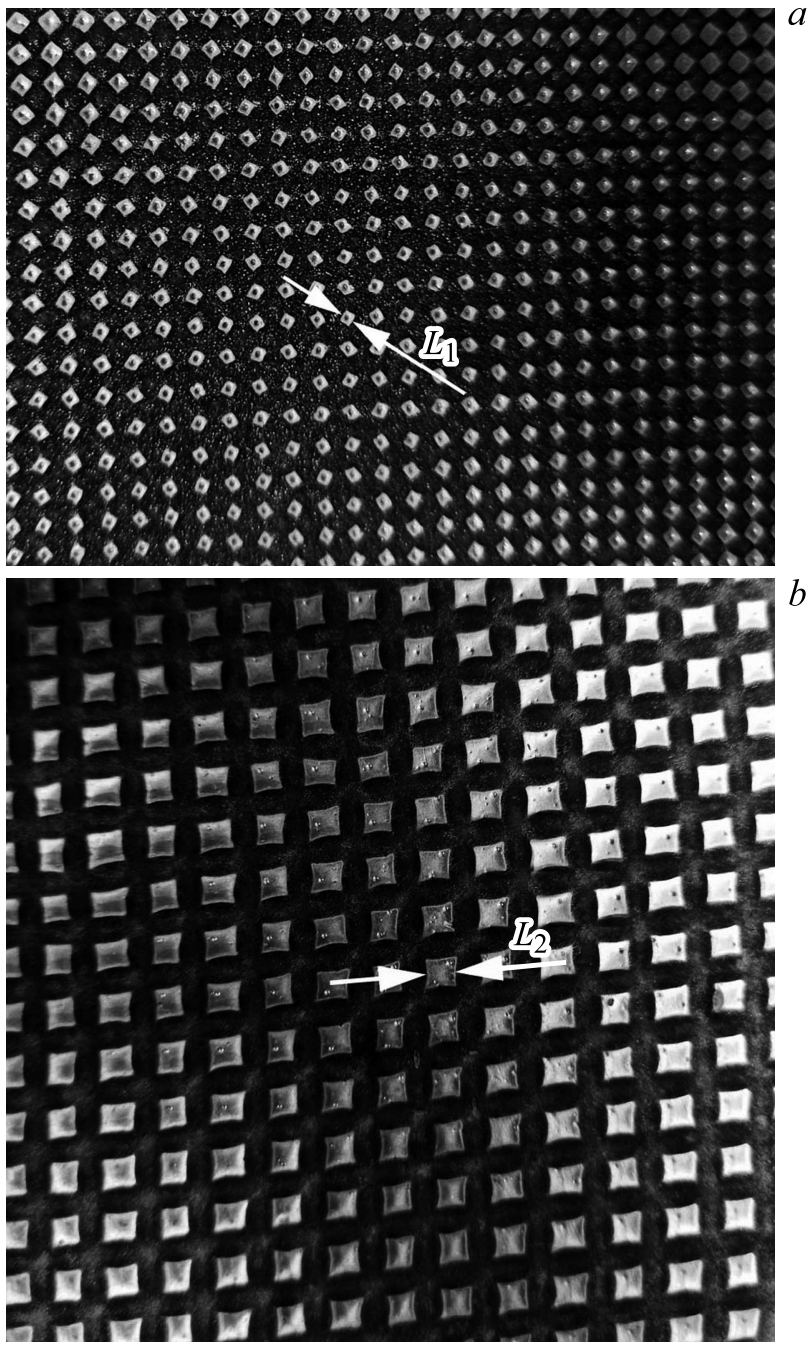

Рис. 5. Изображение рабочих областей на катоде с сетчатым анодом, имеющим размер ячейки: $a-D=1.8 \mathrm{~mm}$ $\left(L_{1}=1 \mathrm{~mm}\right) ; b-D=5 \mathrm{~mm},\left(L_{2}=3 \mathrm{~mm}\right)$. Межэлектродное расстояние $h=1.2 \mathrm{~mm}$.

$r_{D}=9.72 \cdot 10^{-3} \mathrm{~cm}$. Таким образом, объемный заряд не будет влиять на движение заряженных частиц, так как радиус Дебая на несколько порядков меньше длины их свободного пробега $\lambda\left(\lambda_{e}=14.25 \mathrm{~mm}\right.$ - для электронов, $\lambda_{i}=1-2 \mathrm{~mm}$ - для положительных ионов [10]), что говорит о наличии бесстолкновительного слоя относительно большой толщины [16].

\section{Заключение}

Полученная в работе методом конформного отображения модель позволяет построить распределение силовых линий и эквипотенциалей электростатического поля в электродной системе источника внеэлектродной плазмы и оценить влияние размера отверстия в аноде, межэлектродного расстояния, напряжения и толщины анода на распределение электростатического поля. Кроме того, 
модель позволяет проводить расчеты напряженности поля в любой точке моделируемого пространства, а также определять значение энергии и концентрации электронов в зависимости от геометрических параметров электродной системы, что, в свою очередь, может обеспечить оптимизацию технологических процессов (очистка, травление) в зависимости от требуемых характеристик обрабатываемой поверхности.

Все вышесказанное существенно дополняет результаты моделирования, представленные в работах $[9,10]$, и определяет дальнейшее развитие исследований в этом направлении: разработка модели движения заряженных частиц в системе электродов с сетчатым анодом и создание новых приборов, формирующих низкотемпературную внеэлектродную плазму.

Результаты работы хорошо согласуются с экспериментальными данными, что подтверждается как внешним видом катода после длительной работы источника плазмы, так и близкими значениями между концентрациями электронов, рассчитанными с помощью модели и полученными с использованием экспериментальных данных. Таким образом, исходя из таблицы, можно сделать выводы, что для исследуемого источника плазмы более оптимальными геометрическими параметрами электродной системы с точки зрения наибольшей концентрации электронов и равномерности распределения их плотности являются $D=1.8 \mathrm{~mm}, \Delta h=0.5-1 \mathrm{~mm}$, $h=1.2-2.7 \mathrm{~mm}$. В таких случаях концентрация электронов имеет значение $n_{e}=1.27-4.18 \cdot 10^{9} \mathrm{~cm}^{-3}$, что соответствует экспериментальным данным, полученным в работах $[1,3]$.

\section{Конфликт интересов}

Авторы заявляют, что у них нет конфликта интересов.

\section{Список литературы}

[1] Н.Л. Казанский, В.А. Колпаков. Формирование оптического микрорельеба во внеэлектродной плазме высоковольтного газового разряда. (Радио и связь, М., 2009).

[2] В.А. Колпаков, А.И. Колпаков, В.В. Подлипнов. ЖТФ, 83 (4), 41 (2013). [V.A. Kolpakov, A.I. Kolpakov, V.V. Podlipnov. Tech. Phys., 58, (4), 505 (2013).]

[3] N.L. Kazanskiy, V.A. Kolpakov. Optical materials: Microstructuring surfaces with off-electrode plasma. (CRC Press, London, 2017).

[4] И.В. Вагнер, Э.И. Болгов, В.Ф. Гракун, В.Л. Гохвельд., В.А. Кудлай. ЖТФ, 44 (8), 1669 (1974).

[5] В.А. Колпаков. Микроэлектроника, 31 (6), 431 (2002). [V.A. Kolpakov. Russian Microelectronics, $31(6), 431$ (2002).]

[6] Н.Л. Казанский, А.И. Колпаков, В.А. Колпаков. Микроэлектроника, $33(3), 209$ (2004). [N.L. Kazanskii, V.A. Kolpakov, A.I. Kolpakov. Russian Microelectronics, 33 (3), 169 (2004).]
[7] Н.Л. Казанский, В.А. Колпаков. ЖТФ, 79 (9), 41 (2009). [N.L. Kazanskii, V.A. Kolpakov. Tech. Phys., $54(9), 1284$ (2009).]

[8] N.L. Kazanskiy, V.A. Kolpakov, V.V. Podlipnov. Vacuum, 101, 291 (2014).

[9] М.А. Маркушин, В.А. Колпаков, С.В. Кричевский, А.И. Колпаков. ЖТФ, 85 (3), 60 (2015). [M.A. Markushin, V.A. Kolpakov, S.V. Krichevskii, A.I. Kolpakov. Tech. Phys., 60 (3), 376 (2015).]

[10] В.А. Колпаков, С.В. Кричевский, М.А. Маркушин. ЖЭТФ, 151 (1), 189 (2017). [V.A. Kolpakov, S.V. Krichevsky, M.A. Markushin. J. Experiment. Theor. Phys., 124 (1), 164 (2017).]

[11] Н.Н. Миролюбов, М.В. Костенко, М.Л. Левинштейн, Н.Н. Тиходеев. Методы расчета электростатических полей. (Высшая школа, М., 1963).

[12] М.А. Лаврентьев, Б.В. Шабат. Методы теории функиии комплексного переменного. (Наука, М., 1973).

[13] А.Б. Новгородцев, А.Р. Фетхиев, И.С. Фетхиева. Применение функиии комплексного переменного к расчету электростатических полей электродов сложной конфигурации. (Уфимский ордена Ленина Авиационный институт им. Серго Орджоникидзе, Уфа, 1986).

[14] M.A. Markushin, V.A. Kolpakov, S.V. Krichevskiy. CEUR Workshop Proceedings, 1904, 93 (2017). DOI: 10.18287/1613-0073-2017-1904-93-99

[15] Ю.П. Райзер. Физика газового разряда. (Наука, М., 1992).

[16] А.А. Кудрявцев, А.С. Смирнов, Л.Д. Цендин. Физика тлеющего разряда. (Лань, СПб., 2010).

[17] Ю.М. Каган, В.И. Перель. УФН, 81 (3), 409 (1963). DOI: 10.3367/UFNr.0081.196311a.0409 